\title{
Collectivités locales et responsabilité sociale des organisations : quelle impulsion?
}

\section{Catherine Bodet et Annaïg Hache}

\section{(Q) OpenEdition}

Journals

Édition électronique

URL : http://journals.openedition.org/developpementdurable/3104

DOI : 10.4000/developpementdurable.3104

ISSN : 1772-9971

\section{Éditeur}

Association DD\&T

\section{Référence électronique}

Catherine Bodet et Annaïg Hache, «Collectivités locales et responsabilité sociale des organisations : quelle impulsion? », Développement durable et territoires [En ligne], Points de vue (2003-2010), mis en ligne le 08 novembre 2010, consulté le 19 avril 2019. URL : http://journals.openedition.org/ developpementdurable/3104; DOI : 10.4000/developpementdurable.3104

Ce document a été généré automatiquement le 19 avril 2019

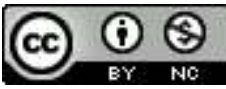

Développement Durable et Territoires est mis à disposition selon les termes de la licence Creative Commons Attribution - Pas d'Utilisation Commerciale 4.0 International. 


\title{
Collectivités locales et responsabilité sociale des organisations: quelle impulsion?
}

\author{
Catherine Bodet et Annaïg Hache
}

1 Si la définition du développement durable proposée en 1987 par le rapport Brundtland (« un développement qui permet de satisfaire les besoins du présent sans compromettre la capacité des générations futures de satisfaire leurs propres besoins ») est très largement admise aujourd'hui, ses interprétations divergent. L'exemple que nous présentons ici, même s'il est limité puisqu'il ne concerne que 5 associations et un territoire, s'appuie sur la volonté de privilégier un développement durable fondé sur une économie plurielle et une "solidarité démocratique ", c'est-à-dire décidée et organisée par le pouvoir politique et la société civile (Laville, 2005).

2 L'exemple de Rennes Métropole est mobilisé dans cet article pour montrer le rôle pivot d'une collectivité locale dans la mise en avant d'une politique de dynamisation de la responsabilité sociale et environnementale sur son territoire. Cette politique s'est appuyée, entre autres, sur l'expérimentation du Bilan Sociétal, outil d'évaluation des pratiques élaboré par le Centre des jeunes dirigeants et acteurs de l'économie sociale (Cjdes) ${ }^{1}$.

3 L'exemple de Rennes est intéressant car il illustre l'idée que la notion de croissance ne règne plus aujourd'hui sans partage et laisse place à d'autres considérations telles que le bien commun et l'utilité sociale. Les attentes actuelles ont une forte dimension immatérielle: "Les demandes des ménages des pays développés ont évolué en liaison avec leur mode de vie et leur mode de pensée. Les attentes en services, d'un côté, les exigences en termes d'environnement et de qualité de vie, de l'autre, ont modifié l'approche de la finalité de la croissance. » (Du Tertre, 2006).

4 Centrée au départ sur la volonté de permettre aux élus de prendre en compte des critères de développement durable dans leur politique d'achat, l'expérimentation du Bilan

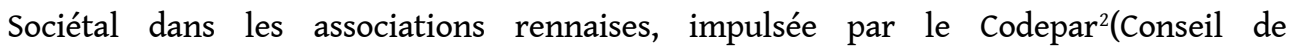


développement économique et social du pays et de l'agglomération de Rennes), vise finalement les coopérations entre les collectivités locales et les associations qu'elles financent dans l'optique d'une contractualisation basée sur un diagnostic partagé et discuté.

Nous resituerons d'abord le contexte local et l'objectif du Codespar de valoriser, d'une part, la contribution de l'économie sociale et solidaire à la vie locale et, d'autre part, de développer un partenariat fondé sur un diagnostic partagé et visant à un nouveau type de contractualisation entre les collectivités locales et les associations. Nous exposerons ensuite le cadre de l'expérimentation et les principaux résultats pour les associations participantes. Nous poserons dans une dernière partie la question de la viabilité du modèle économique de l'évaluation proposé par cette expérimentation. Un tel modèle de cofinancement, partagé entre les collectivités locales et les organisations évaluées, est une des conditions pour que les pratiques d'évaluation de la responsabilité sociale et environnementale se développent dans les petites structures, qui, sans financement spécifique n'y auraient pour la plupart pas accès.

\section{Contexte et but de l'expérimentation}

\subsection{Contexte de l'expérimentation}

6 Le Codespar a expérimenté le Bilan Sociétal dans 5 associations du Pays de Rennes.

7 Sur ce territoire (67 communes et environ 450000 habitants), les associations, mutuelles et coopératives sont nombreuses et leur rôle dans le développement économique est important.

8 Cependant, les capacités de développement de cette économie sont limitées par des facteurs multiples. Certains peuvent être liés à la gestion interne des structures (difficultés à professionnaliser leur intervention, à renouveler le projet, à mobiliser des bénévoles...), d'autres relèvent de la relation entre les structures et leur environnement. Les acteurs rennais ont fait ensemble le constat d'une trop faible visibilité de ce qu'apportent les structures de l'économie sociale et solidaire à la vie locale, entraînant ainsi une certaine banalisation: sont-elles vraiment différentes? Ont-elles une réelle spécificité? Laquelle? Cette spécificité justifie-t-elle un investissement financier spécifique des collectivités publiques?

9 Pour explorer cette question, le Codespar a réalisé une étude ${ }^{3}$ qui aborde ces questions : qu'est-ce qui fait richesse? Qu'est-ce qui fait utilité sociale ? Comment les mesure-t-on? Le Mouvement pour le Développement Solidaire, chargé de cette mission, a réalisé une trentaine d'entretiens avec des responsables associatifs et coopératifs, des techniciens de collectivités ou d'institutions, des habitants impliqués dans des dynamiques locales et des élus locaux. Parmi les constats effectués on peut noter que :

- certaines structures, notamment associatives, ont du mal à mesurer leur action : par manque d'indicateurs rendant compte des différents aspects de leur action, manque de temps, etc.

- les institutions, collectivités locales, administrations partenaires de ces associations disposent également de peu d'outils, de peu de procédures pour mieux saisir les spécificités des associations. 
Ces constats ont amené le Codespar à engager deux démarches participatives locales associant les structures et leur environnement dans la mise en valeur des spécificités des entreprises associatives ${ }^{4}$ :

- la démarche d'expérimentation du bilan sociétal associatif qui est présentée dans le présent article

- Une démarche de mesure de la « valeur ajoutée sociale » dans les associations ${ }^{5}$ : cette démarche vise à faire apparaître, dans la comptabilité associative, l'importance de la contribution nette issue de la sphère du marché, de celle de la collectivité et de celle de la réciprocité associative. La méthode s'appuie sur la valorisation du bénévolat et une nouvelle mise en forme comptable. Cette démarche a été expérimentée auprès de 12 associations.

11 Le Codespar travaille également avec l'ONG PEKEA afin d'avancer sur la définition d'indicateurs de progrès sociétal, avec et pour les gouvernements locaux. Actuellement trois collectivités locales françaises (Rennes Métropole, le Conseil Général d'Ille et Vilaine et le Conseil Régional de Bretagne) sont investies dans la démarche.

\subsection{Les buts recherchés}

Le but de l'expérimentation «bilan sociétal associatif » est d'évaluer et de valoriser les apports de l'économie sociale et solidaire à la vie locale dans une perspective de développement durable et de développement du partenariat entre les associations, les élus et les institutions.

13 L'idée n'est donc pas d'évaluer la politique publique en matière de développement durable, mais de co-construire, entre la collectivité locale et les acteurs associatifs du territoire une politique de développement durable.

\section{Expérimentation du Bilan Sociétal dans les associations rennaises}

\subsection{Le Bilan Sociétal : une évaluation et un processus ${ }^{6}$}

14 Le Bilan Sociétal propose une approche multi-parties prenantes pour engager les structures dans une démarche de progrès, vers une meilleure prise en compte des responsabilités globales de l'organisation envers son milieu.

L'évaluation est réalisée à travers un questionnaire, portant sur 9 domaines ${ }^{7}$, soumis aux différentes parties prenantes internes et externes de l'organisation. Les réponses sont analysées par un intervenant extérieur, labellisé par le Cjdes, à travers 15 critères spécifiques $^{8}$. La pertinence du Bilan Sociétal tient dans le regard croisé des parties prenantes sur la structure. L'évaluation n'est pas réalisée à partir des dires ou de la communication de la direction, mais de l'appréciation croisée des différents acteurs de l'organisation sur son action. C'est en ce sens aussi un outil de partage des informations sur la structure. Les convergences de vues entre les différents acteurs permettent de repérer les points forts de la structure, alors que les divergences de vues pointent d'éventuelles points faibles ou pistes de travail.

La réalisation de l'évaluation fait entrer l'organisation dans un processus de dialogue inter parties prenantes pour l'élaboration d'un diagnostic commun dans un premier 
temps, pour la mise en œuvre de démarches d'amélioration des pratiques dans un second temps.

17 L'évaluation réalisée par le Bilan Sociétal ne donne pas une "note ", ne porte pas de jugement, mais tend à provoquer une mise en mouvement des acteurs sur les thèmes liés à la responsabilité sociale et au développement durable. C'est à cette condition que le processus d'évaluation enrichit l'organisation: il ne s'agit pas d'une «évaluation sanction » ou d'un " évaluation communication », mais d'une « évaluation implication » dans une démarche de progrès.

\subsection{Les apports du Bilan Sociétal associatif : vers une « haute qualité entrepreneuriale »}

Parmi les associations volontaires pour réaliser leur Bilan Sociétal dans le cadre de l'expérimentation impulsée par le Codespar, cinq ont été retenues : l'ADMR de MelesseMontreuil (Aide à domicile en milieu rural), l'Association Rennaise des Centres Sociaux, le CRIJ-Bretagne (Centre d'information jeunesse), les Amitiés sociales (Foyers de jeunes travailleurs), les Compagnons Bâtisseurs Bretagne.

Pour chacune de ces associations, les parties prenantes ont été invitées à répondre au questionnaire du Bilan Sociétal au cours de séances, regroupant une quinzaine de personnes, et animées en deux temps: un temps de renseignement individuel du questionnaire, un temps d'échanges collectifs autour des problématiques soulevées par le questionnaire. Les parties prenantes potentiellement impliquées dans la démarche, selon la pertinence pour chaque association étaient: les administrateurs, les adhérents, les bénévoles, les salariés et leurs représentants, les partenaires et notamment les élus locaux, les clients ou bénéficiaires, les fournisseurs.

Quelques exemples de questions, parmi la centaine posée à tous les acteurs de l'organisation:

- L'association a-t-elle une politique spécifique pour impliquer les jeunes dans le fonctionnement associatif?

- Y-a-t-il une représentation des salariés au sein de conseil d'administration?

- Existe-t-il une clause de " mieux disant social » dans les relations avec les fournisseurs?

- Y-a-t-il une contradiction entre les principes (sociaux, éthiques, moraux...) sur lesquels sont basés les prestations et services de l'association et les contraintes économiques?

- L'association participe-t-elle à des opérations de collecte ou de recyclage des déchets au plan local?

- Dans sa communication externe, l'association fait-elle référence à des valeurs d'intérêt général et d'utilité sociale?...

21 La mise en lumière de l'utilité sociale de l'association était une attente forte des participants vis-à-vis de la méthodologie «Bilan Sociétal ». L'évaluation transversale de l'organisation permet de situer les apports de l'association dans leur contexte, en les positionnant par rapport à un certain nombre de contraintes, d'une part, et de valeurs affichées par les différents acteurs, d'autre part.

Le Bilan Sociétal fournit une appréciation qualitative de l'utilité sociale de l'association. Cette appréciation est portée par une exigence de transparence et une recherche d'objectivité à travers le croisement des réponses des différents acteurs sur la structure à chacune des questions posées. 

fonctionnement et d'organisation des associations participantes. Le rapport, remis à chaque association, souligne les "points forts » sur lesquels l'association peut s'appuyer pour poursuivre son action et met en débat des pistes de progression. Spécifiques à chaque association, les « pistes de travail » portent sur des thèmes très variés, allant de la communication (circulation interne de l'information, communication interne et relation administrateur / salariés, communication auprès des partenaires), au travail sur le projet associatif, en passant par la formation des salariés et les relations fournisseurs ${ }^{9}$.

Le Bilan Sociétal tend à accroître également l'implication des associations sur leur territoire, ainsi que la visibilité de cette implication. Une mise en débat autour de pratiques visant à réduire l'impact des activités sur l'environnement naturel, par exemple, est nécessaire, y compris pour des activités faiblement polluantes: la consommation de papier, les circulations, le recyclage, les habitudes d'achat sont autant de questionnements souvent nouveaux ou en tous cas peu développés et formalisés dans les petites structures.

La mise en débat du rôle et de la connaissance des partenaires publics des associations est apparue comme très importante : quelle est la marge de manœuvre des associations dans le cadre d'un financement lié à l'activité ? Quel type de convention établir avec les partenaires pour concilier les contraintes gestionnaires d'efficacité et d'obligation de résultat avec la responsabilité globale de l'organisation, en accord avec ses valeurs et ses objectifs initiaux?

La mise en mouvement des acteurs internes et externes autour des problématiques du développement durable ouvre à la complexité des questionnements et des arbitrages sans pour autant imposer de voie unique.

\section{3. Évaluation de la responsabilité sociale et environnementale : quel financement ?}

Dans l'expérimentation du Bilan Sociétal associatif réalisé à Rennes, l'évaluation est financée par les collectivités locales, les associations donnant, quant à elles, les moyens techniques et le temps nécessaire à la réalisation de ces évaluations. Elle est réalisée dans un contexte où les collectivités territoriales voient de plus en plus clairement l'intérêt d'évaluer l'action des organisations qu'elles subventionnent et de rendre visible la production de richesses non marchandes.

Nous souhaitons questionner ici ce modèle de répartition des coûts et des charges de travail, dans le cadre d'une interrogation sur la « viabilité économique » de l'évaluation des petites structures. Le financement par les collectivités locales est-il un «modèle économique " possible de l'évaluation des petites structures (associations, PME...) en matière de responsabilité sociale et environnementale ${ }^{10}$

Cette question se pose de plus en plus clairement au fur et à mesure que les problématiques de développement durable s'imposent dans toutes les sphères de l'économie, et ne sont plus réservées aux multinationales, les premières interpellées par la société civile. 
30 Si le coût d'une évaluation n'est pas la seule barrière pour les entreprises, qui redoutent pour certaines de sortir du modèle classique de l'évaluation de la performance, il est parfois cependant discriminant pour les petites entreprises et les associations.

31 L'expérimentation de Rennes démontre la pertinence de l'implication de la collectivité locale dans le financement de l'évaluation des associations. Cette pertinence tient à l'amélioration potentielle de la qualité des prestations offertes à la collectivité, dans la mesure où une évaluation de type "Bilan Sociétal» permet un renforcement des relations et du partenariat entre les associations qui entrent dans la démarche et leurs parties prenantes. Sans financement ou dispositif spécifiques, qui contrecarrent l'inégalité d'accès à l'évaluation et facilitent leur inscription dans une démarche de progrès, les associations seraient exclues de fait des chantiers de l'évaluation.

Il semble plus largement, et au-delà de la prise en charge financière, que la mise en œuvre d'une démarche d'évaluation est facilitée par le caractère collectif de l'opération.

\section{Conclusion}

On retient deux conclusions à approfondir, parmi bien d'autres, pour élargir les perspectives ouvertes par cette expérimentation du Bilan Sociétal :

D'abord, elle montre que les collectivités territoriales peuvent avoir une action politique d'orientation de la RSE, au-delà du champ de l'environnement naturel et de la gestion des effets induits de l'activité économique (infrastructures, formation, gestion des déchets...). Cela montre qu'il est possible pour un territoire de donner une impulsion politique à la RSE, jusqu'ici largement laissée aux seules mains des entreprises.

Ensuite, elle indique la nécessité de continuer à développer de nouveaux outils d'évaluation adaptés aux transformations de la société. Dans une économie de plus en plus guidée par la production immatérielle, on se trouve devant la nécessité de transformer les façons d'évaluer l'économie, et donc de trouver de nouveaux indicateurs, de nouveaux outils, comme le Bilan Sociétal. Cet enjeu doit être resitué dans la crise du capitalisme industriel qui a mis en cause le fordisme depuis une trentaine d'années. Plusieurs éléments caractérisant ce nouveau régime ont des répercussions sur les outils d'évaluation de l'activité des organisations et de leur responsabilité. La crise industrielle induit la montée d'une économie de services qui déstabilise nos manières de produire et de voir la performance des entreprises et des sociétés. La productivité et son contingent de mesures quantifiées ne permettent pas de saisir la complexité de l'offre relationnelle et servicielle, dans les services marchands et plus encore dans les services de proximité. Ces services de proximité sont inscrits dans des politiques territorialisées, et de nouveaux facteurs, qui ne sont plus ni le coût, ni la productivité, ni le volume de production, fondent la performance tant des organisations que des territoires. Plus que la production matérielle finale, ce sont alors les savoirs accumulés (brevets, connaissances...) et la capacité d'organisation qui induisent la performance dans le modèle post-industriel. Le capital immatériel des organisations, qui inclut également des valeurs, du sens (allant de la marque à l'identité institutionnelle en passant par l'identité responsable) ne se saisit pas essentiellement avec des indicateurs quantifiés. 


\section{BIBLIOGRAPHIE}

Catherine Bodet et Dominique Picard, « Le Bilan Sociétal@ : de la prise en compte des intérêts contradictoires des parties prenantes à la responsabilité sociétale », Développement Durable et Territoires, Dossier 5 : Économie plurielle, responsabilité sociétale et développement durable, Mis en ligne le : 10 janvier 2006 ; Disponible sur : http://developpementdurable.revues.org/ document1615.html

Catherine Bodet et Thomas Lamarche, «Le Bilan Sociétal : un processus participatif multi parties prenantes pour la responsabilité sociale des entreprises », in P. de la Broise et T. Lamarche, Responsabilité sociale: Vers une nouvelle communication des entreprises? Presses Universitaires du Septentrion, 2006.

Catherine Bodet, Thomas Lamarche, Gérard Leseul et Dominique Picard, « Évaluation participative dans une économie plurielle. Bilan Sociétal, responsabilité et utilité sociale ", Communication aux VI ${ }^{\mathrm{èmes}}$ Rencontres internationales du Réseau Interuniversitaire de l'Économie Sociale et Solidaire : Économie sociale et solidaire et Europe : quel avenir ?, Grenoble, 1-2 juin 2006. Disponible sur : http://www-sciences-po.upmf-grenoble.fr/IMG/pdf/ bodet_lamarche_leseul_picard.pdf

Michel Capron et Gérard Leseul, "Pour un bilan sociétal des entreprises", Revue des études coopératives et mutualistes et associatives RECMA, n 266, p. 28-41, 1997.

Michel Capron et Françoise Quairel-Lanoizelée, Mythes et réalités de l'entreprise responsable, La Découverte, 2004.

Jean-Louis Laville, « Solidarité et développement durable », in Jean-Pierre Maréchal et Béatrice Quenault (Dir.), Le développement durable. Une perspective pour le XXI ème siècle, PUR, 2005.

Christian du Tertre, « Ouvrir le champ de l'évaluation de la performance au registre des externalités, une condition des coopérations entreprises / collectivités territoriales ", Colloque de Cerisy, Juin 2006 sur le thème : L'économie des services pour un développement durable.

\section{NOTES}

1. Voir Catherine Bodet et Dominique Picard, «Le Bilan Sociétal@ : de la prise en compte des intérêts contradictoires des parties prenantes à la responsabilité sociétale ", Développement Durable et Territoires

Dossier 5 : Économie plurielle, responsabilité sociétale et développement durable, Mis en ligne le : 10 janvier 2006 ; Disponible sur : http://developpementdurable.revues.org/document1615.html 2. Le Codespar rassemble des acteurs socio-économiques (entreprises, syndicats), des élus et des associations. Depuis 2002, il a créé une commission Economie Sociale et Solidaire.

3. Evaluer la richesse et l'utilité sociale dans le pays de Rennes - Etat des lieux préliminaire et perspectives de travail Codespar juin 2004 réalisé par le MDS (Mouvement pour le Développement Solidaire) disponible en ligne : http://www.audiar.org/publications/pdf/emploi/ mds-evaluer-util-sociale.pdf

4. Un colloque intitulé «Apports de l'économie sociale et solidaire au développement. Enjeux et Pratiques de l'évaluation », et organisé par le Codespar et la CRES de Bretagne s'est tenu le 3 
novembre 2005 à Rennes. Ces expérimentations, parmi d'autres, ont été présentées et discutées avec les acteurs locaux.

5. Travail mené en collaboration avec Pascal Perrot, enseignant chercheur à l'Université de Rennes 2 avec le concours de Fanny Corriette ; documents en ligne sur : http://www.audiar.org/ emploi/doc/comptabilite-societale.pdf

Voir également Pascal Perrot, "Une mesure de 'la valeur ajoutée sociale' dans les associations », RECMA n³01, juillet 2006.

6. Voir Catherine Bodet et Thomas Lamarche, « Le Bilan Sociétal : un processus participatif multi parties prenantes pour la responsabilité sociale des entreprises", in P. de la Broise et T. Lamarche, Responsabilité sociale: Vers une nouvelle communication des entreprises? Presses Universitaires du Septentrion, 2006.

7. Produit-services et relations client; Gestion économique; Anticipation-innovationprospective; Organisation du travail et de la production; Gestion des ressources humaines; Acteurs internes de l'entreprise; Environnement humain social et institutionnel; Environnement biophysique ; Finalités-valeurs.

8. Activité, Citoyenneté et participation internes, Citoyenneté externe, Compétitivité, Convivialité, Créativité, Esthétique, Efficacité et efficience, Employabilité et développement des compétences, Éthique, Précaution et prévention, Satisfaction, Solidarité, Utilités sociale et collective, Viabilité.

9. Voir en ligne le tableau de synthèse des résultats du Bilan Sociétal de chaque association : http://www.audiar.org/emploi/doc/bilan-societal.pdf

10. Voir aussi Catherine Bodet, Thomas Lamarche, Gérard Leseul et Dominique Picard, «Évaluation participative dans une économie plurielle. Bilan Sociétal, responsabilité et utilité sociale ", Communication aux VIèmes Rencontres internationales du Réseau Interuniversitaire de l'Économie Sociale et Solidaire : Économie sociale et solidaire et Europe : quel avenir ?, Grenoble, 1-2 juin 2006. http://www-sciences-po.upmf-grenoble.fr/IMG/pdf/ bodet_lamarche_leseul_picard.pdf

\section{RÉSUMÉS}

L'expérimentation du Bilan Sociétal, mise en place par Rennes Métropole dans des associations rennaises, montre comment s'organisent, se pilotent et se financent des initiatives en matière d'évaluation, dans le cadre de l'orientation d'une politique de développement durable et de responsabilité sociale territoriale. Les collectivités locales visent à favoriser de nouvelles formes de contractualisation entre les différents acteurs dans l'optique d'une co-construction d'une politique de développement durable territoriale. Cette expérimentation donne des pistes pour imaginer de nouveaux outils d'évaluation dans le cadre d'une économie plurielle.

This paper presents the Bilan Sociétal experimentation in Rennes associations, realised by Rennes Métropole. It shows that local communities try to favour new forms of contracting between actors in the framework of the sustainable development and local social responsibility policies orientation. It shows however that this experimentation gives tracks to imagine new evaluation tools in a plural economy. 
INDEX

Mots-clés : développement local durable, collectivités locales, responsabilité sociale des organisations

Keywords : local sustainable development, evaluation, bilan sociétal, associations, local communities, corporate social responsibility

\section{AUTEURS}

\section{CATHERINE BODET}

Catherine Bodet économiste, est analyste de la responsabilité sociale et environnementale des organisations, analyste Bilan Sociétal. 19, rue de Lozère - 91400 ORSAY, 0685013773 catherinebodet@wanadoo.fr

\section{ANNAIIG HACHE}

Annaïg Hache, économiste, est chargée de mission « Economie Sociale et Solidaire » au Codespar et Maître de conférences à l'Université Rennes 2 (Master Management des Organisations de l'Economie Sociale et Solidaire) en tant que professionnelle associée. annaig.hache@uhb.fr 